\title{
Chapter 6 \\ Environmental Leadership Development Based on Activity Theory for Sustainable Urban Development in the Greater Pearl River Delta, China
}

\author{
Kyoungjin J. An
}

\begin{abstract}
As cities in the GPRD develop, they go through an environmental transition associated with changes in the type of environmental challenge. With its growing economic power, China is playing an important role in the global economy, although the prosperity of the cities seems to have come with certain hurdles: social inequality and environmental deterioration. In response to such transaction, we have examined the GPRD's urban development, including urban formation, industry relocation, economic development, social inequality, and biodiversity conservation for a better quality of life (QOL). As a catalyst for sustainable urbanization, environmental leadership — where the urbanization process can be in harmony with the urgency for the economic development and sustainable future of the city-was explored. The holistic framework of activity theory was also applied within the area of sustainability to arrive at an inclusive structure for environmental leadership.
\end{abstract}

Keywords Environmental leadership development • Quality of life • Sustainable urban development

\subsection{Introduction}

The Greater Pearl River Delta (GPRD) is a megacity region in southern China, as shown in Fig. 6.1. It includes nine municipalities, which are major component cities of the GPRD in Guangdong Province, and two special administrative regions: Hong Kong and Macao. Currently, the GPRD has a population of over 50 million and

\footnotetext{
K.J. An $(\bowtie)$

Asian Program for Incubation of Environmental Leaders (APIEL),

Department of Urban Engineering, Graduate School of Engineering,

The University of Tokyo, 7-3-1 Hongo, Bunkyo-ku, Tokyo 113-8656, Japan

e-mail: ankj@env.t.u-tokyo.ac.jp
} 


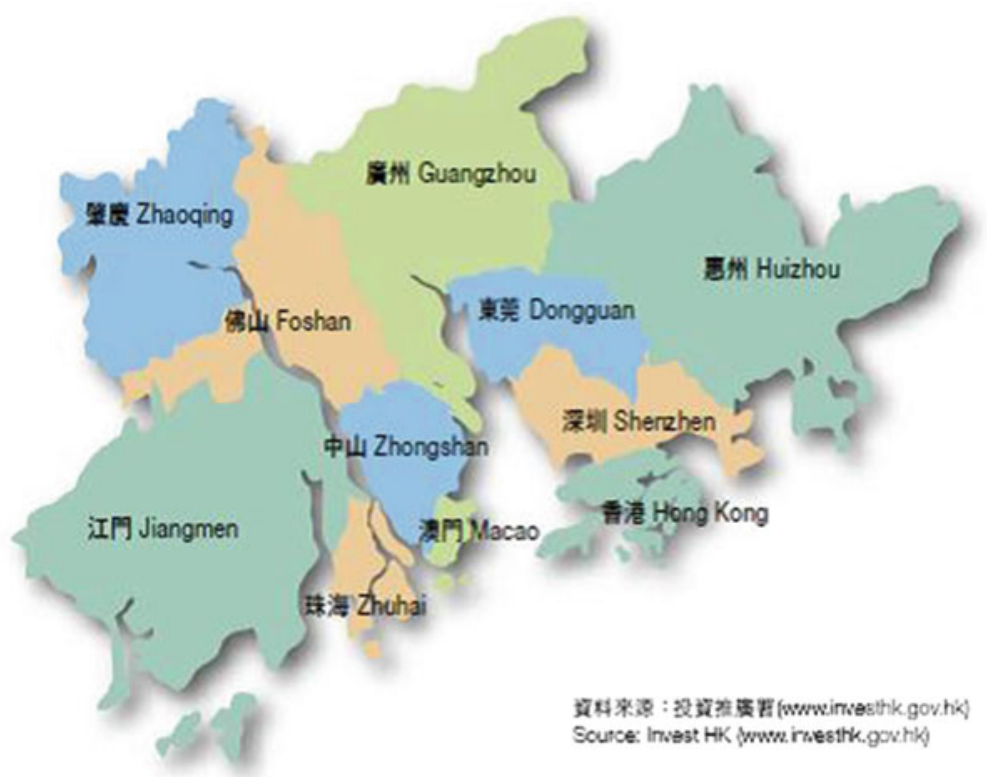

Fig. 6.1 Megacity region of China's Greater Pearl River Delta (GPRD) (Source: Invest HK, www. invest.gov.hk)

covers a land area of around $43,000 \mathrm{~km}^{2}$. About 20 years ago, the GPRD portion in Guangdong Province largely contained underdeveloped rural villages. Since then, there has been an enormous transformation in the GPRD. The "reform and opening up" policy of China has had a dramatic impact on society and the economy over the past 30 years, allowing the country to enter an era of rapid development. Megacity regions, like the GPRD, are one of forerunners in this transformation.

But this rapid change has led to a development that is not only skewed towards economic improvement but it has also created a society with disparities and deprivation. Economically, the GPRD has developed into a renowned world factory, while Hong Kong has been restructured as a regional service center, providing industries in the GPRD with frontend functions, such as research, marketing, and distribution. A regional division of labor, the "front shops, back factories" model began to take shape in the 1990s. Spatially, the GPRD as a whole has become increasingly polycentric; many cities and towns that were formerly peripheral and rural areas have developed into active economic centers. The polycentric spatial form has combined with the rise of urban entrepreneurialism, resulting in a rapidly developing political environment that encourages cities to compete against one another for mobile capital.

The undesirable consequences of political fragmentation are becoming more and more acute. The impacts of Hong Kong and Macao, under the "one country, two systems" model, are added complications to this fragmentation. Political borders stand in the way of coordinated planning. Socially, the GPRD has faced challenges 
caused by an increase in the resident population, which has overwhelmed the GPRD governments. The flood of rural to urban migration has weighed down the infrastructure in cities and has led to tremendous growth of un-serviced urban areas where millions of migrant workers lack access to basic services. There is also a pressing need to address the problems of widespread misuse of land, urban sprawl, traffic congestion, poor sanitation, and the declining living environment in all cities, especially those that are threatened by rapid and often uncontrolled growth, inadequate and poorly maintained infrastructure, industrialization, and the increasing ownership of cars and motorcycles.

Sustainability in urbanization is closely linked with competitiveness especially economic competitiveness [1]. However, competitiveness does not include noneconomic success or accept the consequences, such as social polarization and environmental pollution [2]; favoring economic growth has wider problematic social consequences [3]. Therefore, the concept of competitiveness is being modified to incorporate social and environmental criteria as it directly affects the quality of life. For example, a study by Jiang and Shen [1] suggests that Guangzhou's overall competitiveness among 20 Chinese cities is falling due to the lower social and environmental performance. Perhaps, Guangzhou's competitiveness demands a balance of economic growth with social and environmental performance, which in turn significantly affects the quality of life.

The Asian Program for Incubation of Environmental Leaders (APIEL) of The University of Tokyo (UT) has set out to help understand this balance. As it was introduced in Chap. 1, APIEL is an educational program designed to foster environmental leaders, especially aimed at sustainability issues in Asia. This chapter describes the framework and discusses the circumstances under which activity theory could be used for an environmental leadership program and to help build a better QOL in the cities, within the rapidly urbanizing GPRD region. This field unit took up environmental leadership as a tool for improving the QOL during rapid urbanization as well as sustainable development in the GPRD. In addition, this field unit illustrates the use of environmental leadership for a sustainable future in GPRD cities, using the activity theory framework. Case studies, mostly focusing on major domains for urban QOL, explore the intricate relationship with urbanization. Methods based on activity theory were used to conduct the field unit and manage the data collection and analysis processes. This chapter concludes with a discussion of the relevance and suitability of activity theory as a framework for the current complex problems in fostering future environmental leaders.

\subsection{Activity Theory Framework for Building Leadership Capacity}

Activity theory is a socio-psychological theory with roots in the work of the Russian psychologist Lev Vygotsky during the first half of the twentieth century. Vygotsky's [4] important insight into the dynamics of consciousness was that it is essentially subjective 
and shaped by each person's social and cultural experience. In addition, Vygotsky [4] saw human activity as distinct from non-human entities; it is mediated by tools, the most significant of which is language. Vygotsky's [4] work was continued by others, among them Leont'ey [5], who developed a conceptual framework for a complete theory of human activity. According to Leont'ey [5], activity is a system that has structure, its own internal transitions and transformations, and its own development.

Essentially, Vygotsky [4] defined human activity as a relationship between subject and object, i.e. a person working at something. In this dynamic, purposeful relationship the "always active" subject learns and grows, while the object is interpreted and reinterpreted by the subject in the ongoing conduct of the activity. Approach of activity theory is promising for environmental leadership because it arrives at logical conclusions through a complex interpersonal process of transfer knowledge from individual to group. Engeström [6] gave a more concrete expression to this structure in the triangular representation, which is commonly used to depict an activity. The core of an activity is the relationship between subject (human) and object (purpose) mediated by tools and community. This is a two-way concept of mediation where the capability and availability of tools mediates what is able to be done; the tools, in turn, evolve to hold the historical knowledge of how the community behaves and is organized. The third-generation of activity theory, proposed by Engeström [7] in 2001, advanced the idea of internal contradictions as the driving force for change, diversity and dialogue from divergent perspectives within an activity system, and of networks of interactive activity systems.

Activity theory today is based on the idea that people change or learn when they engage in productive activity, but in productive activity they also change their system. For fostering future leaders, activity theory suggests that leadership occurs through the interaction of the leader with other components of an activity system, such as the tools the leaders have available and the people with whom they interact in a division of labor. Thus, a leader is directed toward a particular goal or outcome. Krasny and Roth [8] explained that any one activity system may overlap with another system, for example, a school and a watershed organization both focus on taking measurements with the goals of producing a report for a class and impacting environmental policy, respectively.

\subsection{GPRD Implementation}

The search for a sustainable future has made fostering environmental leaders essential, especially for mediating between knowledge societies and the community. Studies have shown three behaviors that appear relevant for environmental leadership: articulating an appealing vision with environmental elements, changing perceptions about environmental issues, and taking symbolic action to demonstrate a personal commitment to environmental issues [9]. During the GPRD field exercise, APIEL also emphasizes six attributes for environmental leadership education: information, or the ability to find, understand, and transmit needed information; 
inclusion, or listening and using all available skills and ideas; decision, or defining and pursuing an action agenda; dispatch (action), or doing things now rather than later; standard setting, or formulating the definition of success; and humanity, or using empathy and humor in dealing with others [10].

This chapter will mainly discuss how the students identified a problem and selected a study area for group work during the field exercise provided to practice and build their leadership. The final outcome presented "Sustainable urban development toward Green City: the Greater Pearl River Delta (GPRD), China." in Year 2012 was chosen to describe the challenges during the fieldwork and to reflect the feedback.

\subsubsection{Program Outline}

Over the last 4 years, APIEL has built broad, resonant networks among universities in Asia. The ties with the Hong Kong University of Science and Technology (HKUST) and Sun Yat-sen University (SYSU) grew especially strong by conducting a field exercise called the GRPD Unit over three consecutive years. During the first year of the program (2010), using case studies, we covered environmental issues and the need for environmental leaders in Asia. Broad discussion topics included the environment, the need for environmental leaders, leadership examples in Asia, as well as a sustainable environment and management in the GPRD.

Then, over the next 2 years, the program focused on sustainable urban development in the GPRD. To start, sustainable urban relocation and regeneration of industrial regions in the GPRD were discussed. Since the GPRD is one of the leading economic regions in southern China and a major manufacturing center-combined with the booming economy and Western influences from Hong Kong-it has created an economic gateway attracting foreign capital into mainland China. In this regard, several topics were chosen for students to discuss, and in doing so, build their leadership skills. These topics included trans-boundary issues and collaborative programs to tackle regional air pollution (in turn, to cope with climate change), urban regeneration, and industry relocation for sustainable development in the GPRD.

In the third year, we reviewed the approaches that have been used for the GPRD's urban development, and related environmental loading over the last three decades. An overview of the approaches used in guiding the GPRD's urban development were studied and discussed. In addition, related environmental loading for the past three decades was studied through a lecture and a field trip during the unit. In March 2008, Sir Donald Tsang Yam-kuen, the chief executive of Hong Kong, proposed to the Guangdong party secretary, Wang Yang, that the two territories should jointly form a "Quality Living Circle in the Green Greater Pearl River Delta." The guiding principles would be promoting environmental protection and sustainable development. Table 6.1 summarizes the outline of the field exercise conducted in Year 2012. Students examined GPRD's urban development, including urban formation, industry relocation, economic development, social equity, and biodiversity conservation. 
Table 6.1 Outline of the GPRD Unit field exercise (2012)

Title

Keywords

Date

Place

Participants

Collaborating partners/ organizations

Information lecture

Fieldwork

Group work

Case study area in Guangzhou

Conference presentation
Sustainable urban development toward Green City: the Greater Pearl River Delta (GPRD), China

Environmental leadership development, sustainable urban development, Geographic Information System (GIS), quality of life (QOL)

16-25 February 2012

Hong Kong and Guangzhou, China

24 students from 8 countries-APIEL: 6 students, 3 from the Department of Urban Engineering (UE) and 3 from the Graduate Program in Sustainability Science (GPSS); Hong Kong University of Science and Technology (HKUST): 5 students from engineering; Sun Yat-sen University (SYSU): 7 from science; Seoul National University (SNU): 6 from business, international relations and urban planning

(1) HKUST, SYSU, Asian Institute for Energy, Environment and Sustainability (AIEES) at SNU, and Hong Kong Green Building Council

(1) Urbanization and migration in China; (2) moving to low carbon society in cities; (3) designing high density cities for sustainable and quality living - a few notes for architects, planners and policy makers; (4) environmental GIS applications; (5) global and local environmental loading and quality of life; (6) urban development and sprawl; (7) environmental leadership and GPRD activity theory; (8) green city planning at the city level in the GPRD; (9) city of Guangzhou Urban Land Administration Bureau; (10) rapid urbanization, urban forms and energy consumption in the Pearl River delta; (11) GPRD urban development and its environmental loading; (12) understanding the urban design approach in the process of city planning

(1) Hong Kong Science Park at Shatin; (2) Hong Kong-Shenzhen-Dongguan City Exhibition Hall-Guanzhou SYSU; (3) change of GPRD urban form: Nansha wetland, Greenway in Guangzhou; (4) change of GPRD urban form: Donghaochong, Liwanchong, Shangxiajia, Haizhu Lake; (5) case study area visit and interview/GIS practice

(1) Leadership attributes: communication; (2) GPRD Strengths-WeaknessesOpportunities-Threats (SWOT) analysis, QOL and GIS; (3) develop the indicators for survey questionnaire for quality of life in GPRD; (4) vision and action learning; (5) preparation of final presentation

Industry zone (Honda factory), a higher education mega centre (HEMC, Xiaoguwei island), newly developed town (Zhujiang), well preserved old town (Yangzhong Street village) and an urban village (Xiadu village)

The First International Conference for International Society of Habitat Engineering and Design (ISHED), Asia City in the New Age: (1) Urban Forms and Pro-Environmental Behavior for Waste Management: a Case Study in Guangzhou, China, [11] and (2) Rapid Transformation and Change in Local People's Quality of Life: A Case Study of Xiaoguwei Island, Guangzhou, China [12]

\subsubsection{Applying Activity Theory to GPRD Field Exercise}

As learning activities are dynamic and may have multiple connections among their own elements and with other activity systems, we carefully applied activity theory to design the APIEL education program for urban development in the 


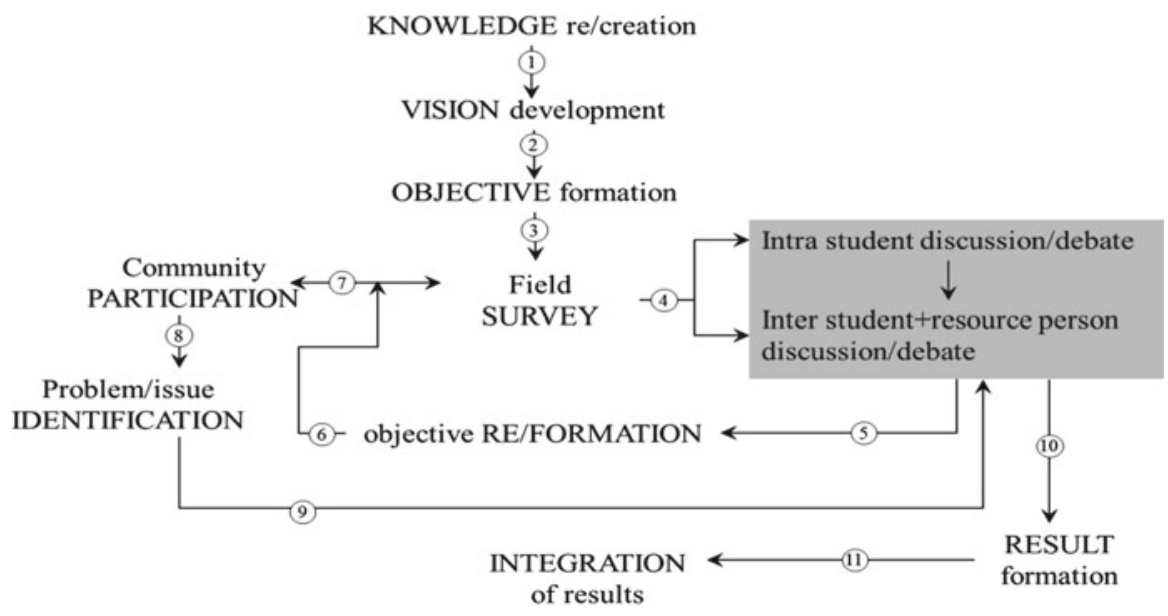

Fig. 6.2 Chronological metrics methodology for leadership development

GPRD. This approach also aided the process of fostering change agents and incubating environmental leadership skills in students. A chronological metrics methodology was developed and is shown in Fig. 6.2. An explicit environmental issue prevalent in Asian cities is used in this leadership education approach. Then, the resource people work with graduate students from multiple disciplines to make explicit the theory and assumptions implicit in the issues they are undertaking. An area is chosen for case studies where the issue is most pressing. After faculty members and students arrive at a consensus about the potential problems and issues, a field unit to the site takes place. A realistic approach is combined with a problem in pre-field unit sessions. Put another way, using the activity theory of change approach, the faculty members seeks evidence that the assumed (or theorized) links between program activities or processes and the desired results can be borne out by the field trips and experience. Faculty members then compare the program theory about how an intervention will unfold with the observations that they make about how it actually did unfold [13].

In February 2012, the APIEL GPRD field exercise undertook field-oriented work on the challenges of QOL in the rapidly urbanizing GPRD region of China. Environmental leadership using an activity theory approach was used for studying the sustainable future of the region. In particular, Guangzhou, where rapid urbanization is taking place, was chosen as the "object" intended to expose students to the scope of problem solving in a city facing severe environmental issues associated with social and economic dimensions. Activity theory and "resonance" were integrated to arrive at final outcomes, as shown in Fig. 6.3.

Students from multiple disciplines and researchers from four premier institutions in Asia-UT, HKUST, SYSU and SNU—playing the role of "subject," came together at Guangzhou to study a sustainable future for the region. Environmental leadership based on ethics, information, and knowledge was extended with tools, such as surveys, geographic information systems (GIS), and divisions of labor 


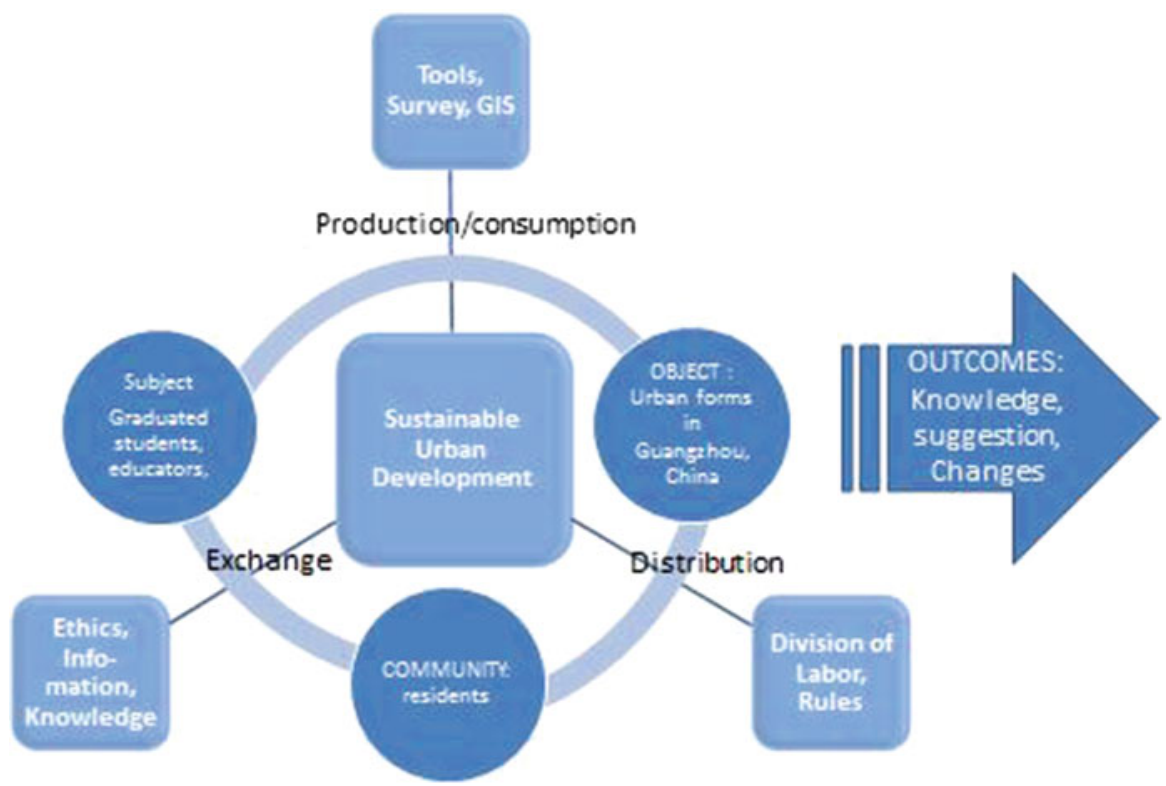

Fig. 6.3 Framework of leadership development for sustainable urban development in Guangzhou

within the program. The final outcome was presented as a report, presentation, and a journal paper.

\subsubsection{The Selection of a Study Area}

Guangzhou is in southern China on the Pearl River Delta plain. The city of Guangzhou has ten districts (Yuexiu, Dongshan, Haizhu, Liwan, Tianhe, Baiyun Whampoa, Fangchun, Huadu and Panyu) with an area of approximately $3,718.8 \mathrm{~km}^{2}$ (about $50 \%$ of the total municipal area) with a census population of 7.4 million (74\% of the total municipal population) [14]. Land use change from 2003 to 2008 in Guangzhou was analyzed using GIS to investigate how urbanization was taking place over a decade.

Dramatic changes in the built-up area over 5 years were compared with other land use, such as water bodies, arable land, transportation, and forests, as illustrated in Fig. 6.4. The built-up area in 2003 is in orange (agglomerated in central Guangzhou); the area in black shows not only the old town but also all over the city as a new town in 2008. It was very clear that the city underwent significant change in land use between 2003 and 2008 due to rapid urbanization. Among the major changes, the most prominent was the increase in built-up area (about $80 \%$ compared with data from 2003). On the other hand, as shown in Fig. 6.5, water bodies and arable land were not conserved but were taken over by built-up areas. 


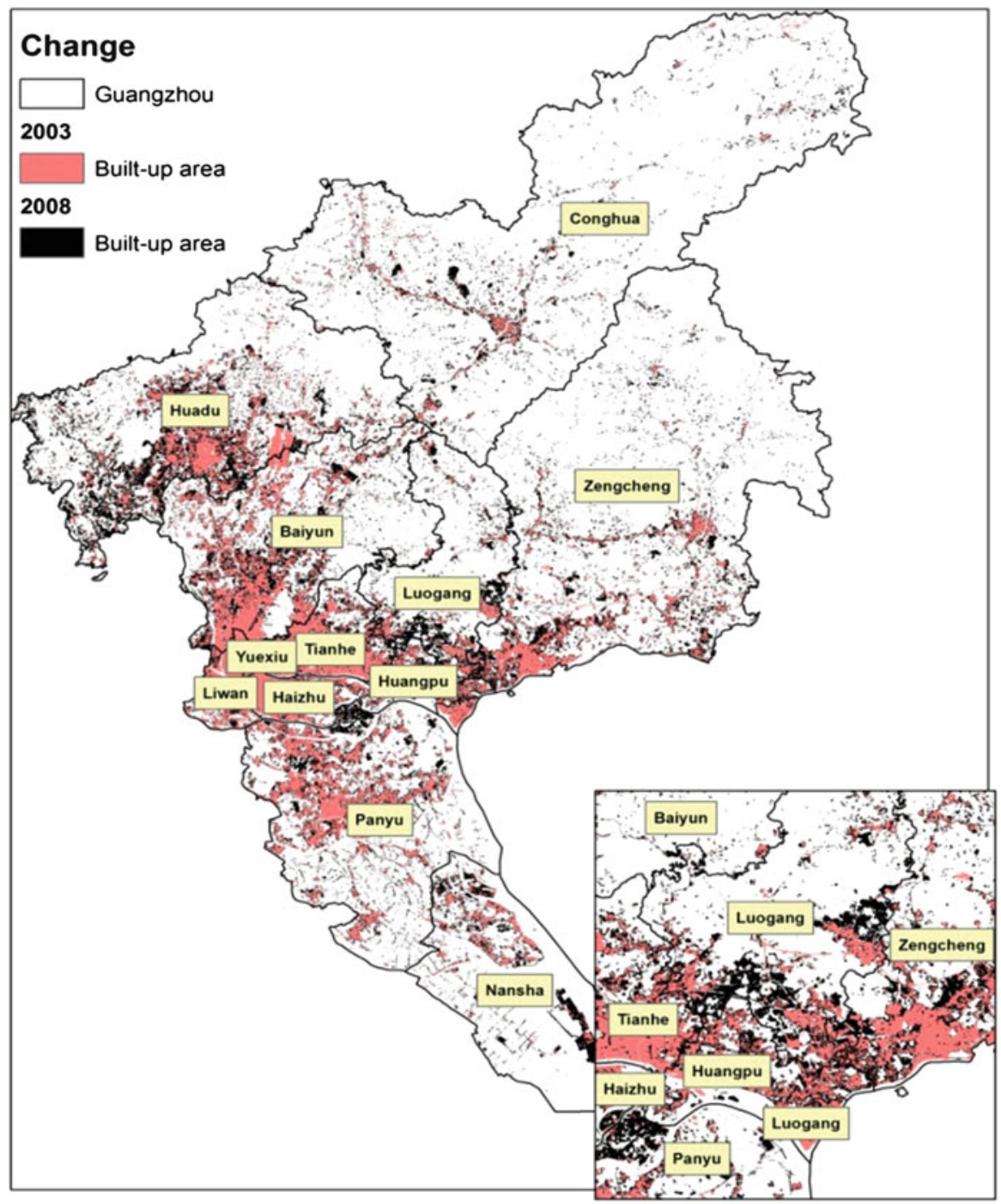

Fig. 6.4 Increase in built-up area Guangzhou from 2003 to 2008

Mindful of this urban development, we conducted empirical studies in the Guangzhou to investigate QOL using survey-based approaches to assess a sustainable method for urban development. The survey, including objective and subjective data, was integrated with a GIS tool to enhance the investigation of QOL. Students selected a study area and did group work on site. To begin with, the city of Guangzhou, as a study field, was divided into five areas according to their urban form: industry zone (Honda factory), a higher education mega centre (Xiaoguwei island), newly developed town (Zhujiang), well-preserved old town (Yangzhong 


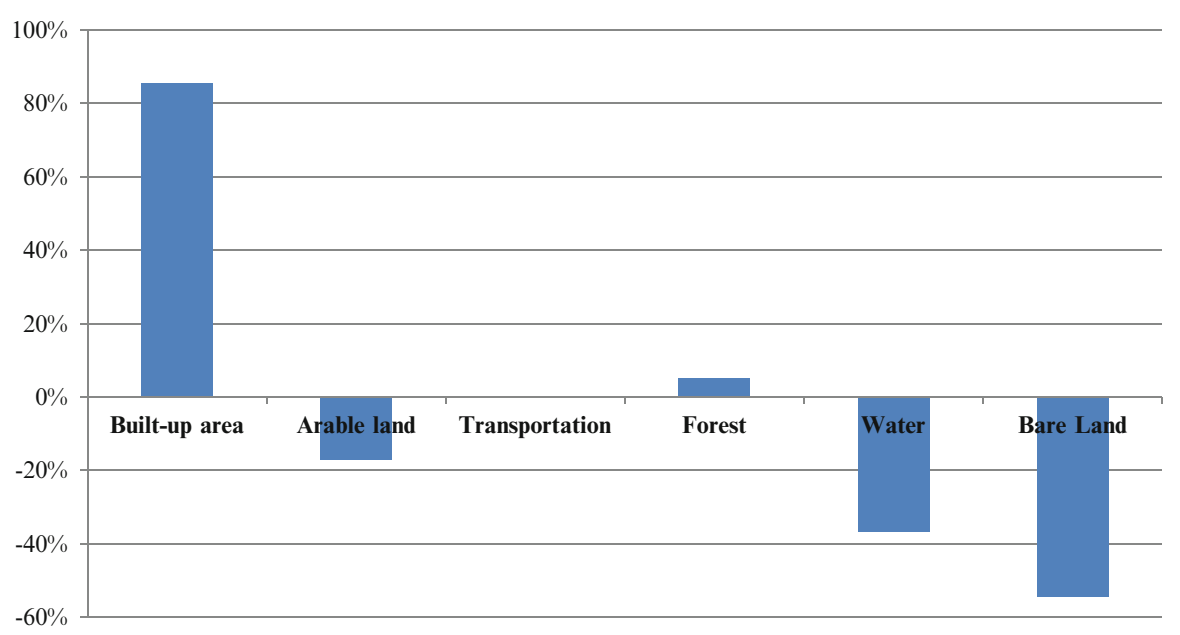

Fig. 6.5 Land use change (\%) in Guangzhou from 2003 to 2008

Street village), and an urban village (Xiadu village) for newcomers seeking economic opportunities, as shown in Fig. 6.6.

The selection of a study area for each group was based on the vision created by the group members through communication, knowledge creation, and objectives. This process was challenging because students from different backgrounds, countries, major subjects of study, and personal characteristics had to build trust through exchange and then divide up roles, as illustrated in Fig. 6.3. Through the lens of QOL, each urban form for Guangzhou's urban development was measured and extended to the pro-environmental behavior of the residents. Learning occurred through the interaction of the learner with the components of social sustainability in the field. The creation of a vision, division of labor, and generating a knowledge base through reconnaissance and sample surveys, were used for arriving at particular policy-level outcomes.

\subsubsection{Creating Vision: Challenges from Student's Feedback}

This section was abstracted from student reports expressing the challenges they faced during the unit. First, most students said that arriving at a consensus for a common goal was a challenge. A student wrote in her final report that the group's decision on the vision was a spontaneous process of continuous discussion, and that a systematic division of roles was the next step in the process. In her group, vision creation was done through personal interest, then a tree of functions was drawn and every member of the team was asked to write their names beside their choice for work. This approach was then later adopted as a common methodology. Postanalysis and brainstorming were used in her group to focus on waste management 

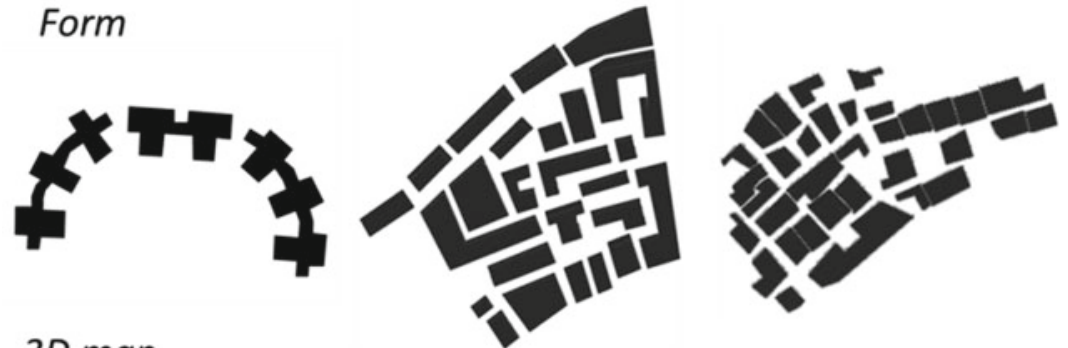

3D map
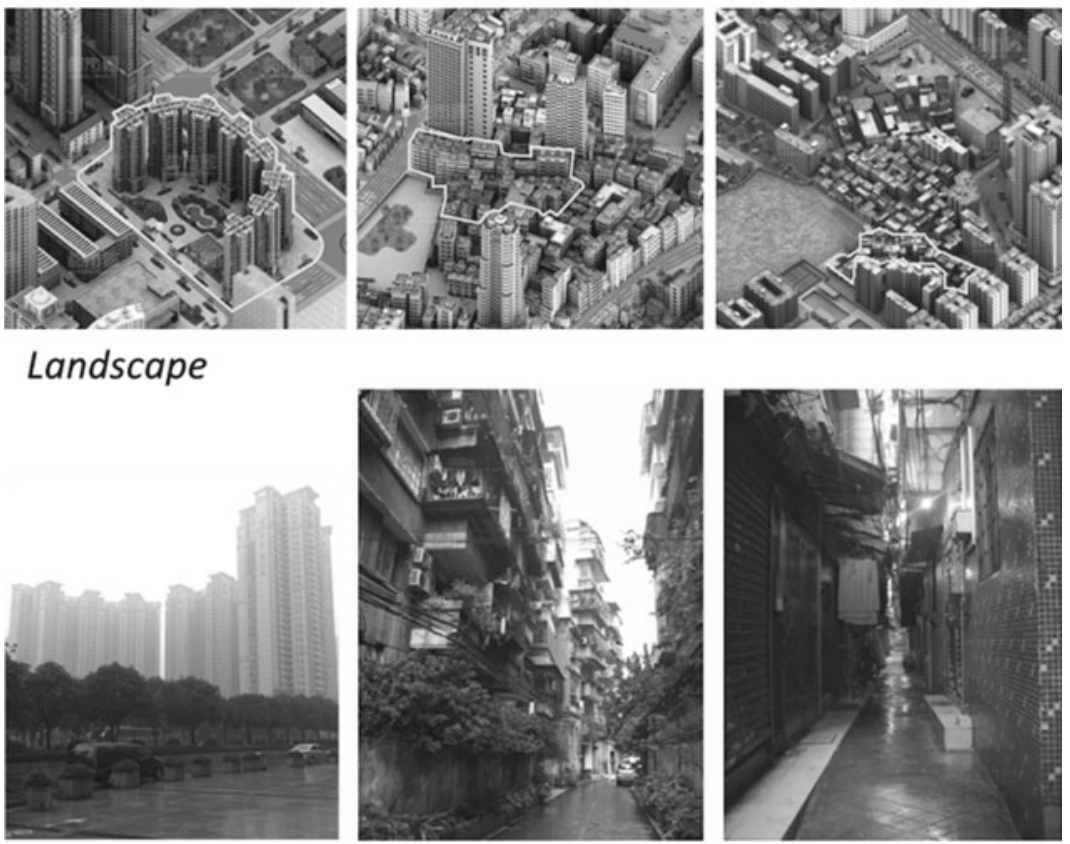

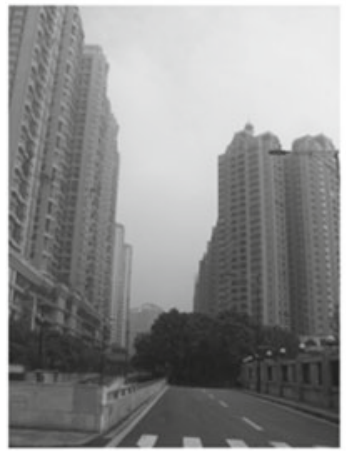

(a) New Town

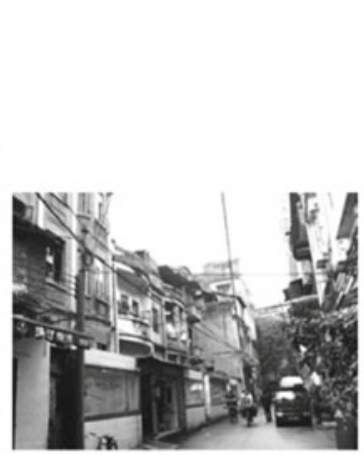

(b) Old Town



(c) Urban Village

(Source of 3D maps: http://gz.o.cn)

Fig. 6.6 Urban forms in Guangzhou [11] 
and the pro-environmental sector. Another student also emphasized this challenge, saying that "after the first reconnaissance survey, we understood that the major challenge which we faced was to narrow down the study objectives, as we all found issues were myriad." After deciding on the focus, sketching out the framework and choosing a methodology for the study were found to be easy and his group proceeded smoothly. Students addressed important findings through activities, saying, "From the beginning we tried to remain focused on the objectives that we were trying to tackle. At times, we did deviate from our main point of view, but with guidance from resource persons, we got back on track. Importance of multi-disciplinary approach was such an essential tool; we wouldn't have realized that before." Whenever his group understood the gaps that each one of them had, they then tried to identify the potential among the team. Then all members tried to maximize the quality of output through patient listening, discussing, debating, and taking notes. To begin with, members differed on the shared vision. Students also mentioned that each of the members in her group had a different focus of concern at the beginning of the program, so they spent quite a long time on group discussion but could not arrive at a common vision. However, more knowledge about Guangzhou during the lectures and fieldwork helped them make a breakthrough in vision creating. All groups had members from different countries and academic backgrounds.

Communication and mutual understanding were found essential to work with people from diverse backgrounds and to arrive at a common vision. Eventually, the students came together on the object of the study and worked together, despite. Sustainable urban development as a catalyst of leadership capacity building in this program involves many factors, including the environment, economics, and society. At the same time, many stakeholders' viewpoints have to be considered.

\subsubsection{Challenges from Educators for Appraisal}

The success of this program was evaluated in two ways: the report and/or paper students produced based on the outcome of the program and a self-evaluation of leadership. As this program emphasizes not only an environmental problem solving but also the process of handling and solving them, and in so doing develop leadership, it is challenging to measure how much the students developed their leadership skills. Faculty members have asked students at the end of program: How did you create a shared vision with others? What was the problem(s) you and your group members identified? What was the strategy you built up to solve them? Did you create a vision table, information needs diagram, group matrix, action agenda, and implementation plan? Did those lead you to an integrated problem solution? Did you commit to being a standard setter? Did you positively study and practice essential leadership attributes? Did you find the areas of your strength through participation in this program? Did you find the areas of your weakness through participation in this program? and How are you going to apply your leadership skills when you are an environmental leader in the future? Table 6.2 shows the self-evaluations of leadership after the unit from six UT 
Table 6.2 Self-evaluation of leadership after the unit from UT students (six students)

\begin{tabular}{|c|c|c|c|c|}
\hline & & $\begin{array}{l}\text { Standard } \\
\text { met }\end{array}$ & $\begin{array}{l}\text { Standard met } \\
\text { with concerns }\end{array}$ & $\begin{array}{l}\text { Standard } \\
\text { not met }\end{array}$ \\
\hline (1) & $\begin{array}{l}\text { Creating a clear vision to solve an } \\
\text { environmental problem }\end{array}$ & 5 & & 1 \\
\hline (2) & Applying information to solve the problem & 5 & 1 & \\
\hline (3) & $\begin{array}{l}\text { Developing communicating, listening, } \\
\text { and interpersonal skills }\end{array}$ & 6 & & \\
\hline (4) & Use of resources and facilities & 4 & 2 & \\
\hline (5) & $\begin{array}{l}\text { Decision: ability to define and pursue } \\
\text { an action agenda }\end{array}$ & 5 & 1 & \\
\hline (6) & $\begin{array}{l}\text { Action: ability to take on tasks NOW, } \\
\text { rather than later }\end{array}$ & 5 & 1 & \\
\hline (7) & Collaborating with others & 6 & & \\
\hline (8) & Sense of humor during the unit & 5 & 1 & \\
\hline (9) & Understanding lectures and fieldwork & 4 & 2 & \\
\hline$(10)$ & Overall achievement & 5 & 1 & \\
\hline
\end{tabular}

students. Though we found that one student couldn't meet the standard for creating a clear vision to solve an environmental problem, communication and collaboration standards were met by all students. This implies that among the leadership attributes that we have taught, inclusion was found important in our program.

For the academic achievement, the two presentations listed below were made at international conferences:

Md. Manjur Morshed, Kyoungjin J. An, Xu Xiaohan, Hironori Hamasaki, Tomomi Hoshiko (2012) Rapid Transformation and Change in Local People's Quality of Life: A Case Study of Xiaoguwei Island, Guangzhou, China. The 1st International Conference on Habitat Engineering and Design, October 13-14, 2012, Tongji University, China. Oral Presentation.

Jungchan Lee, Kyoungjin An (2012) Urban Forms and Pro-Environmental Behavior for Waste Management: a Case Study in Guangzhou of China, The 1st International Conference on Habitat Engineering and Design, October 13-14, 2012, Tongji University, China. Oral Presentation.

\subsection{Conclusions}

Environmental leadership in this context is about personal growth or change within a group to guide positive development toward a vision of an environmentally friendly and better future. Our belief, based on many years of professional experience, is that leadership can be learned. Indeed, we think that all participants who joined the GPRD Unit will lead others at some time and place in their career, and that overt preparation for leadership should be an integral part of professional education and experience. Many traditional leaders are given high marks for 
accomplishing much in a short time, and moreover, have been first and foremost, effective adversaries of competing causes; slaying dragons has always been a sure route to leadership success. However, in this chapter we argue that environmental problems are not most effectively solved through processes. Finding common ground, negotiation, and cooperation are better suited to most of the complex, longterm problems facing environmental leaders.

This chapter examined how educators, students, and other people in higher academic settings can work together to build transformational leadership capacity to help drive more sustainable urban development practices. In relation to universitybased higher education, we linked customized leadership development activities (based on activity theory) with prominent urbanization issues in Guangzhou. This provided a system to produce educational reports, papers, and conferences. The process of building knowledge and skills as well as social networks, then finally led to behavioral change in a variety of proposals presented by students. Thus, this chapter argues that building transformational leadership capacity within a higher academic setting is another way educators can lead our society to desirable behavioral change and sustainable urban development. Such leadership development initiatives are essential in contemporary environmental learning, and they can be both meaningful and worthwhile.

This indicates that the APIEL can go alongside a socially critical approach, but more importantly, it focuses on experience where fieldwork takes place within an activity system. We have observed how students assimilate an approach that reflects the complexity of thinking and learning in relation to the environment. Feedback from multiple sources was collected and used to evaluate and improve the activity. This research is both ongoing and challenging.

Acknowledgments The GPRD Unit was successfully conducted with solid support from Sun Yat-sen University and Hong Kong University of Science and Technology, and special thanks are extended to Prof. Shiyu Li at SYSU and Prof. Guanghao Chen at HKUST for their strong support.

Open Access This article is distributed under the terms of the Creative Commons Attribution Noncommercial License which permits any noncommercial use, distribution, and reproduction in any medium, provided the original author(s) and source are credited.

\section{References}

1. Jiang Y, Shen J (2010) Measuring the urban competitiveness of Chinese cities in 2000. Cities 27:307-314

2. Lever WF, Turok I (1999) Competitive cities: introduction to the review. Urban Stud 36:791-794

3. Wang J (2009) Art in capital: shaping distinctiveness in a culture-led urban regeneration project in Red Town, Shanghai. Cities 26:245-254

4. Vygotsky LS (1978) Mind in Society: the development of higher psychological processes. Harvard University Press, Cambridge 
5. Leont'ey AN (1981) Problem of the development of the mind. Progress, Moscow

6. Engeström Y (1996) Developmental work research as educational research, Nordisk Pedagogik. J Nordic Educ Res 16(5):131-143

7. Engeström Y (2001) Expansive learning at work: toward an activity theoretical reconceptualization. J Educ Work 14(1):133-156

8. Krasny ME, Roth WM (2010) Environmental education for social-ecological system resilience: a perspective from activity theory. Environ Educ Res 16:545-558

9. Portugal E, Yuki G (1994) Perspectives on environmental leadership. Leadersh Q 5:271-276

10. Gordon JC, Berry KJ (2006) Environmental leadership equals essential leadership. Yale University Press, New Haven

11. Lee JC, An KJ (2012) Urban forms and pro-environmental behavior for waste management: a case study in Guangzhou of China. In: The 1st international conference on habitat engineering and design, Oral presentation, Tongji University, China, 13-14 October 2012

12. Morshed MM, An KJ, Xu XH, Hamasaki H, Hoshiko T (2012) Rapid transformation and change in loc.l people's quality of life: a case study of Xiaoguwei Island, Guangzhou, China. In: The 1st international conference on habitat engineering and design, Oral presentation, Tongji University, China, 13-14 October 2012

13. Weitzman BC, Silver D, Dillman KN (2002) Integrating a comparison group design into a theory of change evaluation: the case of the urban health initiative. Am J Eval 23(4):371-385

14. Xu J, Yeh AGO (2003) City profile: Guangzhou. Cities 20:361-374 